\title{
Stimulatory Effect of the Magnetic Treatment on the Germination of Cereal Seeds
}

\author{
E. Martinez, M. Florez, M.V. Carbonell \\ Department of Agroforestry Engineering. Physics section. School of Agricultural, Food and Biosystems Engineering. Ciudad \\ Universitaria s/n 28040 Madrid, Spain
}

\begin{abstract}
The main objective of this study is to determine the effects of $125 \mathrm{mT}$ magnetic treatment on the germination of different cereals seeds. This objective has a practical application in agriculture science: early growth of seeds. Germination tests were carried out under laboratory conditions by exposing seeds to magnetic field for different times. For each treatment the number of germinated seeds was counted to determine the time necessary to achieve the final maximum percentage of germinated seeds; rate of germination was assessed by determining the mean germination time (MGT) and parameters T1, T10, T25, T50, T75, T90, time required to germinate 1 - 90 percent of seeds. An increase in the percentage and rate of germination of seeds as positive response to magnetic field treatment in rice, wheat, maize and barley seeds have been found for all treatments applied. The mean germination time and parameters were reduced for all the magnetic treatments applied. Most significant differences were obtained for time of exposure of chronically exposure and 24 hours. External magnetic fields enhance seed vigor by influencing the biochemical processes by stimulating activity of proteins and enzymes. Numerous studies suggested that magnetic field increases ions uptake and consequently improves nutrition value.
\end{abstract}

Keywords-cereal seeds, magnetic field, germination, seedling, magnet.

\section{INTRODUCTION}

The effects on living systems of exposure to a magnetic field, particularly on germination of seeds and growth of plants, have been the object of much research. In general, the enhancement of growth due to magnetic field exposure appears to have been confirmed by many scientists. Some have tried to determine effects related with seed germination, such as changes in biochemical activity, curvature, magnetotropism and germination rate. The main objective of this study is to determine the effects of magnetic treatment, in addition to the geomagnetic field, on the germination of cereal seeds.

Scientists have found an induction of primary root curvature in radish seedlings in a static magnetic field, roots responded tropically to magnetic field with the tropism appearing to be negative; these roots responded significantly to the south pole of the magnet (Yano, et al. 2001). The application of magnetic field doses of $4 \mathrm{mT}$ and $7 \mathrm{mT}$ promoted germination ratios of bean and wheat seeds (Cakmak, et al. 2010). Magnetic treated pea plants grew higher and heavier than control; the greatest differences were observed for seeds treated with doses of $125 \mathrm{mT}$ and $250 \mathrm{mT}$, for 24 hours or permanent. Germination curves show the earlier germination process and higher germination rate; curves of treated seeds are lightly displaced towards the left side rate. Furthermore, the final percentage of seeds is higher than control seeds (Carbonell et al. 2011). The positive effects on plants characteristics such as seed germination rate, shoot development, length and weight of plants and yield is reported by numerous authors.

\section{MATERIAL AND METHODS}

Germination tests of cereal seeds were carried out under laboratory conditions with natural light and the minimum and maximum temperature of $18{ }^{\circ} \mathrm{C}$ and $22^{\circ} \mathrm{C}$, according to guidelines issued by the International Seed Testing Association (ISTA, 2004) with only slight modifications. Cereal seeds (maize, rice, barley and wheat) were supplied by the Spanish Office of Vegetable Varieties, which guarantees high seed viability and homogeneity and thus significant results with smaller samples.

\subsection{Magnetic treatment}

Magnetic treatment consisted of different doses (D) due to variation in exposure time $(\mathrm{t})$ and magnetic field induction (B). The static magnetic field was generated by permanent ring magnets, with $125 \mathrm{mT}$ strength, external diameter of $7.5 \mathrm{~cm}$, internal diameter of $3 \mathrm{~cm}$, and height of $1 \mathrm{~cm}$. Ring analogous to the ring magnets, of the same material but without magnetic induction, were used as blind (Control). Magnetic doses were obtained by exposing the seeds to each magnetic field for different times: from 1 minute to chronically exposure. The experimental design involves four replicates $(n=4)$ with 25 seeds. Thus, groups of 100 seeds were subjected to each magnetic treatment, and an analogous group was used as control. In each replicate the Petri dishes with seeds was placed on magnet for the corresponding time: $1 \mathrm{~min}$ D1, $10 \mathrm{~min}$ D2, $20 \mathrm{~min}$ 
D3, 1 hour D4 24 hours D5 and for all experimental period or chronical exposure D6.

\subsection{Germination test}

The goal of this test was to determine the possible influence of magnetic treatment on the time required for germination. Germination was tested by placing 25 treated seeds per Petri dish around a circular line, on filter papers soaked with $12 \mathrm{ml}$ of distilled water. Petri dishes with seeds were labeled and randomly located. Experimental groups D1-D6 and control C ran simultaneously. For each treatment the number of germinated seeds was counted to determine the time necessary to achieve the final maximum percentage of germinated seeds $\left(G_{\max }\right)$. Seeds were considered germinated when their radicle measured at least $1 \mathrm{~mm}$. The rate of germination was assessed by determining the mean germination time (MGT) and time required to germinate $1,10,25,50,75$ and 90 percent of seeds (parameters $\mathrm{T}_{1}, \mathrm{~T}_{10}, \mathrm{~T}_{25}, \mathrm{~T}_{50}, \mathrm{~T}_{75}$ and $\mathrm{T}_{90}$ ).

\subsection{Statistical analysis}

Statistical analysis of variance and mean comparisons $(\mathrm{p} \leq 0.001 ; 0.001 \leq \mathrm{p} \leq 0.01 ; 0.01 \leq \mathrm{p} \leq 0.05)$ was performed using the Seedcalculator software developed for seed germination data analysis by Plant Research International; the software provides the germination curves for each treatment, a comparison of the results of all the treatments and a comparison of those results with the result of the control.

\section{RESULTS}

Figures 1-4 show the germination curves for each treatment and control: fig. 1 for maize, fig. 2 for rice, fig. 3 for barley and fig. 4 for wheat. Treatment curves are placed to left side that indicates the diminution of germination parameters $T_{1}-T_{90}$, consequently the higher rate of germination of cereals seeds. Curves also show the great percentage of germination.

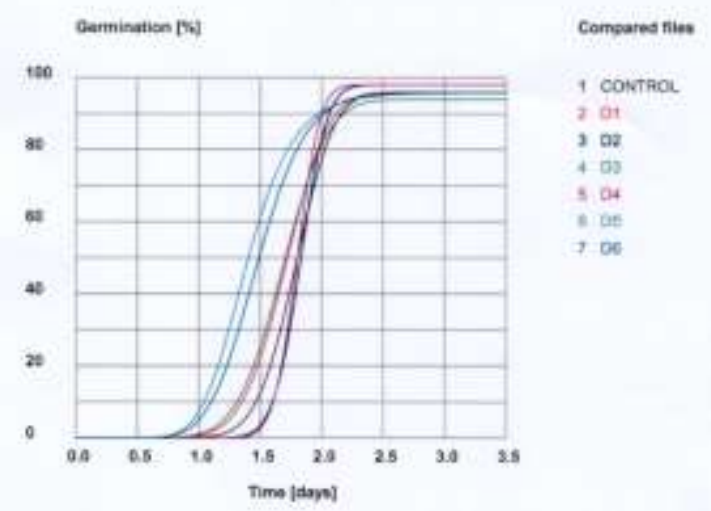

Fig.1: Germination curves of maize seeds subjected to 125 mT magnetic field. Doses D1-D6 versus control C.

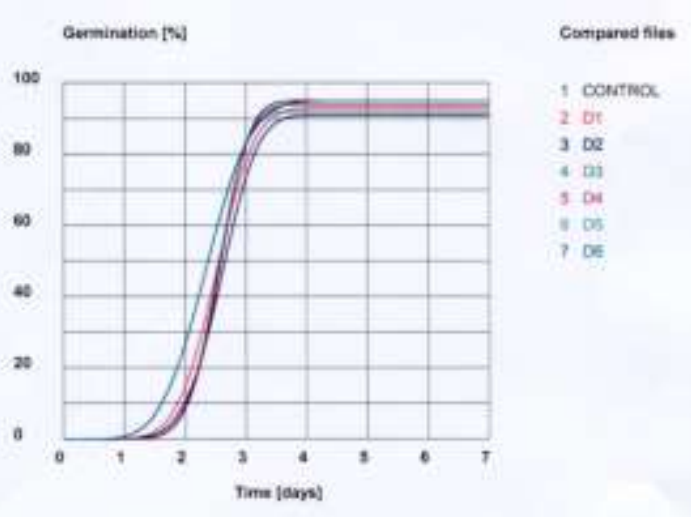

Fig.2: Germination curves of rice seeds subjected to 125 mT magnetic field. Doses D1-D6 versus control C.

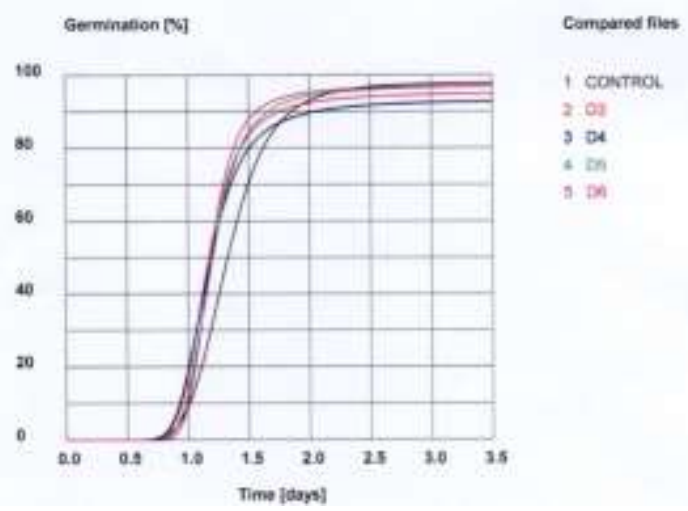

Fig.3: Germination curves of barley seeds subjected to 125 mT magnetic field. Doses D1-D6 versus control C.

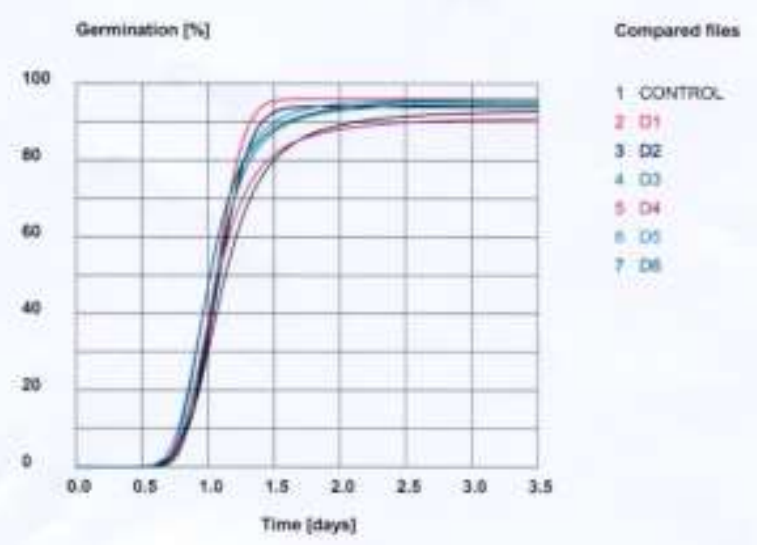

Fig.4: Germination curves of wheat seeds subjected to 125 mT magnetic field. Doses D1-D6 versus control C.

In germination test, percentage of germinated seeds $\left(\mathrm{G}_{\max }\right)$ and the time required for germination (parameters MGT, $\mathrm{T}_{1}-\mathrm{T}_{90}$ ) were determined for each dose, expressed as the mean of the four replicates and their standard error; the germination parameters obtained from cereals seeds exposed to $125 \mathrm{mT}$ magnetic field (doses D1-D6), are provided in Table 1 for maize, Table 2 for rice, Table 3 for barley and Table 4 for wheat seeds. The number of 
germinated seeds $\left(\mathrm{G}_{\max }\right)$, from 80 to $99 \%$, corroborates the high quality of seeds. Fewer than $90 \%$ of seeds exposed to a magnetic field germinated earlier than the control seeds; parameters $\mathrm{T}_{10}-\mathrm{T}_{90}$ and the mean germination time were reduced for all the applied magnetic doses.

Table.1: Germination parameters for maize seeds subjected to $125 \mathrm{mT}$ stationary magnetic field for exposure times: 1 minute (D1), 10 minutes (D2), 20 minutes (D3), 60 minutes (D4), 24 hours (D5), chronic exposure (D6) and control $(C)$. $G_{\text {max }}$ : number of germinated seeds (\%); MGT: Mean germination time; $T_{1}, T_{10}, T_{25}, T_{50}, T_{75}, T_{90}$ : time needed to obtain 1, 10, 25, 50, 75 and $90 \%$ of seeds to germinate, expressed in hours. Asterisks show significant differences whit control $(C): * * * *$ extremely significant $(p<0.001)$; *** very significant $(0.001<p<0.01)$; **significant $(0.01<p<0.05)$.

\begin{tabular}{|c|c|c|c|c|c|c|c|c|}
\hline \multirow[b]{2}{*}{$\begin{array}{c}\text { Parameter } \\
\text { D }\end{array}$} & \multirow[b]{2}{*}{$G_{\max }(\%)$} & \multicolumn{7}{|c|}{ Time (hours) $\bar{x} \pm$ standard error } \\
\hline & & $\mathbf{T}_{1}$ & $\mathbf{T}_{10}$ & $\mathbf{T}_{25}$ & $\mathbf{T}_{\mathbf{5 0}}$ & $\mathbf{T}_{75}$ & $\mathbf{T}_{90}$ & MGT \\
\hline \multirow[t]{2}{*}{$\mathbf{C}$} & 96.00 & 28.56 & 35.28 & 39.12 & 43.20 & 47.52 & 51.84 & 42.72 \\
\hline & \pm 2.31 & \pm 2.40 & \pm 1.92 & \pm 1.44 & \pm 0.96 & \pm 0.72 & \pm 12.48 & \pm 0.96 \\
\hline \multirow[t]{2}{*}{ D1 } & 96.00 & 25.20 & 32.4 & 36.72 & 41.28 & 46.08 & 50.88 & 40.08 \\
\hline & \pm 0.00 & \pm 2.16 & \pm 1.92 & \pm 1.68 & \pm 1.44 & \pm 0.96 & \pm 0.72 & \pm 1.44 \\
\hline \multirow[t]{2}{*}{ D2 } & 98.00 & 33.84 & 38.40 & 41.04 & 43.92 & 46.80 & 49.44 & 43.68 \\
\hline & \pm 1.15 & \pm 1.68 & $\begin{array}{c} \pm 1.20 \\
* *\end{array}$ & $\begin{array}{c} \pm 0.96 \\
* *\end{array}$ & \pm 0.72 & \pm 0.48 & \pm 0.48 & \pm 0.72 \\
\hline \multirow[t]{2}{*}{ D3 } & 94.00 & 24.00 & 31.44 & 36.00 & 41.04 & 46.32 & 52.32 & 40.32 \\
\hline & \pm 1.15 & $\begin{array}{c} \pm 1.20 \\
* *\end{array}$ & $\begin{array}{c} \pm 0.96 \\
* *\end{array}$ & $\begin{array}{c} \pm 0.72 \\
* * *\end{array}$ & $\begin{array}{c} \pm 0.48 \\
* * *\end{array}$ & $\begin{array}{c} \pm 0.24 \\
* *\end{array}$ & \pm 0.72 & $\begin{array}{c} \pm 0.48 \\
* * *\end{array}$ \\
\hline \multirow[t]{2}{*}{ D4 } & 98.00 & 34.56 & 38.64 & 40.08 & 43.20 & 45.84 & 48.24 & 43.20 \\
\hline & \pm 1.15 & $\begin{array}{c} \pm 0.48 \\
* *\end{array}$ & \pm 0.48 & \pm 0.48 & \pm 0.48 & $\begin{array}{c} \pm 0.24 \\
* *\end{array}$ & \pm 0.24 & \pm 0.48 \\
\hline \multirow[t]{2}{*}{ D5 } & 96.00 & 19.20 & 24.96 & 28.80 & 33.84 & 39.60 & 47.52 & 34.32 \\
\hline & \pm 2.31 & $\begin{array}{c} \pm 1.44 \\
* * *\end{array}$ & $\begin{array}{l} \pm 0.24 \\
* * * *\end{array}$ & $\begin{array}{l} \pm 0.96 \\
* * * *\end{array}$ & $\begin{array}{l} \pm 1.68 \\
* * * *\end{array}$ & $\begin{array}{l} \pm 2.40 \\
* * * *\end{array}$ & \pm 3.12 & $\begin{array}{l} \pm 0.96 \\
* * * * *\end{array}$ \\
\hline \multirow[t]{2}{*}{ D6 } & 96.00 & 19.92 & 26.40 & 30.72 & 35.76 & 41.32 & 48.48 & 35.76 \\
\hline & \pm 2.31 & $\begin{array}{c} \pm 1.68 \\
* * *\end{array}$ & $\begin{array}{c} \pm \mathbf{0 . 4 8} \\
* * * *\end{array}$ & $\begin{array}{c} \pm \mathbf{0 . 4 8} \\
* * * *\end{array}$ & $\begin{array}{c} \pm 1.20 \\
* * * *\end{array}$ & $\begin{array}{c} \pm 1.44 \\
* * * *\end{array}$ & \pm 3.36 & $\begin{array}{l} \pm 0.24 \\
* * * * *\end{array}$ \\
\hline
\end{tabular}

Table.2: Germination parameters for rice seeds subjected to $125 \mathrm{mT}$ stationary magnetic field for exposure times: 1 minute (D1), 10 minutes (D2), 20 minutes (D3), 60 minutes (D4), 24 hours (D5), chronic exposure (D6) and control (C). $G_{m a x}$ : number of germinated seeds (\%); MGT: Mean germination time; $T_{1}, T_{10}, T_{25}, T_{50}, T_{75}, T_{90}$ : time needed to obtain 1, 10, 25, 50, 75 and $90 \%$ of seeds to germinate, expressed in hours. Asterisks show significant differences whit control $(C): * * * *$ extremely significant $(p<0.001)$; *** very significant $(0.001<p<0.01)$; **significant $(0.01<p<0.05)$.

Parameter

Time (hours) $\bar{x} \pm$ standard error

\begin{tabular}{|c|c|c|c|c|c|c|c|c|}
\hline Dose & $G_{\max }(\%)$ & $\mathbf{T}_{1}$ & $\mathbf{T}_{10}$ & $\mathbf{T}_{25}$ & $\mathbf{T}_{50}$ & $\mathbf{T}_{75}$ & $\mathbf{T}_{90}$ & MGT \\
\hline C & $\begin{array}{r}95.00 \\
\pm 1.91\end{array}$ & $\begin{array}{l}37.92 \\
\pm 1.44\end{array}$ & $\begin{array}{l}48.96 \\
\pm 0.96\end{array}$ & $\begin{array}{l}55.20 \\
\pm 0.72\end{array}$ & $\begin{array}{r}62.16 \\
\pm 0.48\end{array}$ & $\begin{array}{l}69.12 \\
\pm 0.24\end{array}$ & $\begin{array}{r}76.56 \\
\pm 0.96\end{array}$ & $\begin{array}{c}61.20 \\
0.72\end{array}$ \\
\hline D1 & $\begin{array}{l}93.00 \\
\pm 1.91\end{array}$ & $\begin{array}{c}32.40 \\
\pm 3.60 \\
* *\end{array}$ & $\begin{array}{c}45.12 \\
\pm 2.64 \\
\quad * *\end{array}$ & $\begin{array}{c}52.80 \\
\pm 1.44 \\
* *\end{array}$ & $\begin{array}{r}61.44 \\
\pm 0.96\end{array}$ & $\begin{array}{c}70.56 \\
\pm 0.96 \\
* *\end{array}$ & $\begin{array}{c}82.08 \\
\pm 2.64 \\
* * *\end{array}$ & $\begin{array}{r}60.00 \\
\pm 0.72\end{array}$ \\
\hline D2 & $\begin{array}{r}91.00 \\
\pm 4.43\end{array}$ & $\begin{array}{r}35.04 \\
\pm 3.84\end{array}$ & $\begin{array}{c}47.76 \\
2.88\end{array}$ & $\begin{array}{l}55.20 \\
\pm 1.92\end{array}$ & $\begin{array}{r}63.60 \\
\pm 1.20\end{array}$ & $\begin{array}{l}72.72 \\
\pm 0.72 \\
* * * *\end{array}$ & ------- & $\begin{array}{r}61.92 \\
\pm 0.96\end{array}$ \\
\hline D3 & $\begin{array}{c}92.00 \\
\pm 1.00 \\
* *\end{array}$ & $\begin{array}{l}37.92 \\
\pm 2.40\end{array}$ & $\begin{array}{l}48.48 \\
\pm 1.68\end{array}$ & $\begin{array}{l}54.72 \\
\pm 1.20\end{array}$ & $\begin{array}{r}61.68 \\
\pm 0.48\end{array}$ & $\begin{array}{r}68.88 \\
\pm 0.48\end{array}$ & $\begin{array}{c}78.72 \\
\pm 1.20 \\
* *\end{array}$ & $\begin{array}{r}60.24 \\
\pm 0.72\end{array}$ \\
\hline
\end{tabular}




\begin{tabular}{|c|c|c|c|c|c|c|c|c|}
\hline D4 & $\begin{array}{r}94.00 \\
\pm 2.00\end{array}$ & $\begin{array}{r}37.44 \\
\pm 3.60\end{array}$ & $\begin{array}{c}48.48 \\
2.64\end{array}$ & $\begin{array}{r}54.96 \\
\pm 1.92\end{array}$ & $\begin{array}{r}61.92 \\
\pm 0.96\end{array}$ & $\begin{array}{r}69.12 \\
\pm 0.48\end{array}$ & $\begin{array}{r}77.28 \\
\pm 2.64\end{array}$ & $\begin{array}{r}60.96 \\
\pm 1.20\end{array}$ \\
\hline D5 & $\begin{array}{r}95.00 \\
\pm 1.91\end{array}$ & $\begin{array}{l}25.68 \\
\pm 1.44 \\
* * * *\end{array}$ & $\begin{array}{c}38.88 \\
\pm 1.20 \\
* * * *\end{array}$ & $\begin{array}{c}47.28 \\
\pm 0.96 \\
* * * *\end{array}$ & $\begin{array}{l}57.12 \\
\pm 0.48 \\
* * * *\end{array}$ & $\begin{array}{c}67.68 \\
\pm 0.72 \\
* *\end{array}$ & $\begin{array}{r}79.20 \\
\pm 3.36\end{array}$ & $\begin{array}{l}56.40 \\
\pm 0.48 \\
* * * *\end{array}$ \\
\hline D6 & $\begin{array}{r}95.00 \\
\pm 1.91\end{array}$ & $\begin{array}{l}24.96 \\
\pm 1.68 \\
* * * *\end{array}$ & $\begin{array}{c}38.64 \\
\pm 1.68 \\
* * * *\end{array}$ & $\begin{array}{l}47.28 \\
\pm 1.44 \\
* * * *\end{array}$ & $\begin{array}{l}57.12 \\
\pm 0.96 \\
* * * *\end{array}$ & $\begin{array}{c}67.20 \\
\pm 0.96 \\
* *\end{array}$ & $\begin{array}{r}78.48 \\
\pm 3.12\end{array}$ & $\begin{array}{l}56.16 \\
\pm 1.20 \\
* * * *\end{array}$ \\
\hline
\end{tabular}

Table.3: Germination parameters for barley seeds subjected to $125 \mathrm{mT}$ stationary magnetic field for exposure times: 1 minute (D1), 10 minutes (D2), 20 minutes (D3), 60 minutes (D4), 24 hours (D5), chronic exposure (D6) and control (C). $G_{\text {max }}$ : number of germinated seeds (\%); MGT: Mean germination time; $T_{1}, T_{10}, T_{25}, T_{50}, T_{75}, T_{90}$ : time needed to obtain 1, 10 , 25, 50, 75 and $90 \%$ of seeds to germinate, expressed in hours. Asterisks show significant differences whit control $(C): * * * *$ extremely significant $(p<0.001)$; *** very significant $(0.001<p<0.01)$; **significant $(0.01<p<0.05)$.

Parameter

Time (hours) $\bar{x} \pm$ standard error

\begin{tabular}{|c|c|c|c|c|c|c|c|c|}
\hline Dose & $\mathbf{G}_{\max }(\%)$ & $\mathbf{T}_{1}$ & $\mathbf{T}_{10}$ & $\mathbf{T}_{25}$ & $\mathbf{T}_{50}$ & $\mathbf{T}_{75}$ & $\mathbf{T}_{90}$ & MGT \\
\hline $\mathbf{C}$ & $\begin{array}{c}98 \\
\pm 1.15\end{array}$ & $\begin{array}{l}19.44 \\
\pm 0.96\end{array}$ & $\begin{array}{l}24.24 \\
\pm 0.96\end{array}$ & $\begin{array}{c}27.6 \\
\pm 0.72\end{array}$ & $\begin{array}{r}31.92 \\
\pm 0.72\end{array}$ & $\begin{array}{l}37.20 \\
\pm 0.48\end{array}$ & $\begin{array}{r}44.40 \\
\pm 0.96\end{array}$ & $\begin{array}{r}33.12 \\
\pm 0.48\end{array}$ \\
\hline D1 & $\begin{array}{c}96 \\
\pm 1.63\end{array}$ & $\begin{array}{l}19.68 \\
\pm 0.48\end{array}$ & $\begin{array}{l}24.24 \\
\pm 0.48\end{array}$ & $\begin{array}{l}27.12 \\
\pm 0.48\end{array}$ & $\begin{array}{r}31.20 \\
\pm 0.48\end{array}$ & $\begin{array}{c}36.24 \\
\pm 0.24 \\
* *\end{array}$ & $\begin{array}{l}44.16 \\
\pm 0.72\end{array}$ & $\begin{array}{c}31.92 \\
\pm 0.48 \\
* *\end{array}$ \\
\hline D2 & $\begin{array}{c}100 \\
\pm 0.00\end{array}$ & $\begin{array}{l}19.44 \\
\pm 0.72\end{array}$ & $\begin{array}{l}23.52 \\
\pm 0.24\end{array}$ & $\begin{array}{c}26.16 \\
\pm 0.48 \\
* *\end{array}$ & $\begin{array}{c}29.76 \\
\pm 0.96 \\
* *\end{array}$ & $\begin{array}{c}34.32 \\
\pm 1.68 \\
* *\end{array}$ & $\begin{array}{c}40.56 \\
\pm 2.64 \\
* *\end{array}$ & $\begin{array}{c}31.44 \\
\pm 1.20 \\
* *\end{array}$ \\
\hline D3 & $\begin{array}{c}95 \\
\pm 1.91\end{array}$ & $\begin{array}{l}19.44 \\
\pm 0.72\end{array}$ & $\begin{array}{c}22.80 \\
\pm 0.48 \\
* *\end{array}$ & $\begin{array}{c}24.96 \\
\pm 0.72 \\
* * *\end{array}$ & $\begin{array}{l}28.60 \\
\pm 0.96 \\
* * * *\end{array}$ & $\begin{array}{l}32.88 \\
\pm 1.20 \\
* * * *\end{array}$ & $\begin{array}{r}42.00 \\
\pm 2.16\end{array}$ & $\begin{array}{r}29.52 \\
\pm \mathbf{0 . 9 6} \\
* * * *\end{array}$ \\
\hline D4 & $\begin{array}{c}93 \\
\pm 1.91\end{array}$ & $\begin{array}{l}18.72 \\
\pm 0.96\end{array}$ & $\begin{array}{c}22.32 \\
\pm 0.72 \\
* *\end{array}$ & $\begin{array}{c}24.96 \\
\pm 0.72 \\
* * *\end{array}$ & $\begin{array}{l}28.56 \\
\pm 0.72 \\
* * * *\end{array}$ & $\begin{array}{c}34.08 \\
\pm 1.20 \\
* * *\end{array}$ & ------ & $\begin{array}{r}29.76 \\
\pm \mathbf{0 . 4 8} \\
* * * *\end{array}$ \\
\hline D5 & $\begin{array}{c}97 \\
\pm 1.91\end{array}$ & $\begin{array}{l}20.16 \\
\pm 0.72\end{array}$ & $\begin{array}{l}23.52 \\
\pm 0.24\end{array}$ & $\begin{array}{c}25.68 \\
\pm 0.24 \\
* * *\end{array}$ & $\begin{array}{l}28.80 \\
\pm 0.48 \\
\text { **** }\end{array}$ & $\begin{array}{l}33.12 \\
\pm 1.20 \\
* * * *\end{array}$ & $\begin{array}{c}39.84 \\
\pm 3.60 \\
* *\end{array}$ & $\begin{array}{r}30.00 \\
\pm 0.24 \\
* * * *\end{array}$ \\
\hline D6 & $\begin{array}{c}97 \\
\pm \mathbf{1 . 0 0}\end{array}$ & $\begin{array}{c}21.12 \\
\pm 0.72 \\
* *\end{array}$ & $\begin{array}{l}24.00 \\
\pm 0.24\end{array}$ & $\begin{array}{c}25.92 \\
\pm 0.24 \\
* * *\end{array}$ & $\begin{array}{r}28.56 \\
\pm 0.48 \\
* * * *\end{array}$ & $\begin{array}{l}31.92 \\
\pm 0.24 \\
* * * *\end{array}$ & $\begin{array}{r}37.68 \\
\pm 1.92 \\
* * * *\end{array}$ & $\begin{array}{r}29.52 \\
\pm 0.48 \\
* * * *\end{array}$ \\
\hline
\end{tabular}

Table.4: Germination parameters for wheat seeds subjected to $125 \mathrm{mT}$ stationary magnetic field for exposure times: 1 minute (D1), 10 minutes (D2), 20 minutes (D3), 60 minutes (D4), 24 hours (D5), chronic exposure (D6) and control (C). $G_{\text {max: }}$ number of germinated seeds (\%); MGT: Mean germination time; $T_{1}, T_{10}, T_{25}, T_{50}, T_{75}, T_{90}$ : time needed to obtain $1,10,25$, 50, 75 and $90 \%$ of seeds to germinate, expressed in hours. Asterisks show significant differences whit control (C): **** extremely significant $(p<0.001)$; *** very significant $(0.001<p<0.01)$; **significant $(0.01<p<0.05)$.

Parameter

\begin{tabular}{|c|c|c|c|c|c|c|c|c|c|c|}
\hline \multirow{2}{*}{ Dose } & $\mathbf{G}_{\max }(\boldsymbol{\%})$ & $\mathbf{T}_{\mathbf{1}}$ & $\mathbf{T}_{\mathbf{1 0}}$ & $\mathbf{T}_{\mathbf{2 5}}$ & $\mathbf{T}_{\mathbf{5 0}}$ & $\mathbf{T}_{\mathbf{7 5}}$ & T90 & MGT \\
\hline $\mathbf{C}$ & 93.00 & 16.32 & 20.40 & 23.28 & 27.12 & 33.60 & 51.12 & $\mathbf{2 8 . 8}$ \\
& \pm 1.91 & \pm 0.96 & \pm 0.72 & \pm 0.48 & \pm 0.48 & \pm 0.96 & \pm 11.28 & $\mathbf{\pm 0 . 7 2}$ \\
\hline
\end{tabular}

Time (hours) $\bar{x} \pm$ standard error 


\begin{tabular}{|c|c|c|c|c|c|c|c|c|}
\hline $\mathbf{D 1}$ & 96.00 & 16.08 & 20.16 & 22.80 & 25.68 & 28.56 & 31.68 & $\mathbf{2 5 . 4 4}$ \\
& \pm 1.63 & \pm 0.48 & \pm 0.48 & \pm 0.48 & \pm 0.24 & \pm 0.24 & \pm 0.24 & $\pm \mathbf{0 . 4 8}$ \\
& & & & & $* * *$ & $* * * *$ & & $* * * *$ \\
\hline $\mathbf{D 2}$ & 94.00 & 15.36 & 19.92 & 22.80 & 26.16 & 29.52 & 33.84 & $\mathbf{2 5 . 6 8}$ \\
& \pm 1.15 & \pm 0.48 & \pm 0.48 & \pm 0.48 & \pm 0.48 & \pm 0.24 & \pm 3.60 & $\mathbf{\pm 0 . 2 4}$ \\
& & & & & $* *$ & $* * * *$ & & $* * * *$ \\
\hline $\mathbf{D 3}$ & 95.00 & 17.04 & 20.16 & 22.56 & 25.68 & 30.00 & 39.12 & $\mathbf{2 6 . 8 8}$ \\
& \pm 2.52 & \pm 0.72 & \pm 0.48 & \pm 0.24 & \pm 0.24 & \pm 1.20 & \pm 3.36 & $\mathbf{\pm 0 . 4 8}$ \\
& & & & & $* * * *$ & $* * *$ & $* * *$ & $* * *$ \\
\hline $\mathbf{D 4}$ & 91.00 & 16.08 & 19.68 & 22.32 & 25.92 & 32.16 & & $\mathbf{2 7 . 1 2}$ \\
& \pm 3.42 & \pm 0.48 & \pm 0.48 & \pm 0.24 & \pm 0.48 & \pm 1.68 & ---- & $\mathbf{0 . 9 6}$ \\
& & & & $* *$ & $* * *$ & & & $* *$ \\
\hline $\mathbf{D 5}$ & 97.00 & 15.12 & 18.48 & 21.12 & 24.48 & 29.04 & 36.48 & $\mathbf{2 5 . 4 4}$ \\
& \pm 1.91 & \pm 0.24 & \pm 0.24 & \pm 0.24 & \pm 0.24 & \pm 0.48 & \pm 1.68 & $\mathbf{\pm 0 . 7 2}$ \\
& $* *$ & $* *$ & $* * *$ & $* * * *$ & $* * * *$ & $* * * *$ & $* * * *$ & $* * * *$ \\
\hline $\mathbf{D 6}$ & $\mathbf{9 8 . 0 0}$ & $\mathbf{1 6 . 0 8}$ & $\mathbf{1 9 . 2 0}$ & $\mathbf{2 1 . 3 6}$ & $\mathbf{2 4 . 4 8}$ & $\mathbf{2 9 . 2 8}$ & $\mathbf{3 8 . 6 4}$ & $\mathbf{2 5 . 9 2}$ \\
& $\pm \mathbf{1 . 1 5}$ & $\pm \mathbf{0 . 4 8}$ & $\pm \mathbf{0 . 2 4}$ & $\pm \mathbf{0 . 2 4}$ & $\pm \mathbf{0 . 2 4}$ & $\pm \mathbf{0 . 9 6}$ & \pm 2.40 & $\mathbf{\pm 0 . 4 8}$ \\
& $* * *$ & & $* *$ & $* * * *$ & $* * * *$ & $* * * *$ & $* * * *$ & $* * * *$ \\
\hline
\end{tabular}

Mean germination time (MGT) of control seeds was significantly reduced for most doses. Time required to germinate $1 \%$, parameter $\mathrm{T}_{1}$, of seeds exposed to a magnetic field was less than control. As time required for germinate $1 \%$ of seeds, $T_{1}$, is closely related to the onset of germination, these results indicate that cereals seeds exposed to a magnetic field sprouted earlier. The time required for germination recorded for each treatment was, in general, less than the corresponding control values; thus the rate of germination of treated seeds was higher than that of the untreated seeds (C). Results of maize germination show extremely significant differences for doses D5 and D6 in mean germination time and $\mathrm{T}_{1}-\mathrm{T}_{75}$ parameters; significant differences were also founded for doses D2, D3 and D4. Germination curves obtained for all doses are displaced towards left side rate. The onset of rice germination shows significant differences for D1 and extremely for TMG and $\mathrm{T}_{1}-\mathrm{T}_{50}$ parameters; the same behavior of germination curves were observed. Table 3 shows the significant differences observed for barley in D2-D6 doses, thus, the germination rate was improved.

Wheat results are similar of other cereal seeds, a higher germination rate was obtained for magnetic doses applied; best results were obtained for D6, D5. The results obtained in this study are in concordance with preliminary researches carried out for authors in grass, legumes, medicinal and others seeds.

\section{DISCUSSION}

Although the mechanisms at work in plants and other living systems exposed to a magnetic field are still not well known, several theories have been proposed, including biochemical changes or altered enzyme activities (Phirke, et al. 1996). An experimental study on water absorption by lettuce seeds previously exposed to a stationary magnetic field of $1-10 \mathrm{mT}$ was carried out; an increase in water uptake due to the applied magnetic field, which could explain of the increase in the germination speed of treated lettuce seeds was reported (Gracía et al. 2001). Exposure to magnetic fields improved parameters like water uptake, leaf photosynthetic efficiency and leaf protein content was found in soybean Shiene et al. 2011). Our results are in agreement with the germination data of maize seeds, an increase in germination and shoot development in seeds exposed to $150 \mathrm{mT}$ magnetic field for 10, 15, 20 and 30 minutes was found (Aladjadjiyan, 2002). Magnetic treatment of $30 \mathrm{mT}$ and $85 \mathrm{mT}$ on two broad bean cultivars affected positively the germination and emergence (Podlesni et al. 2004). The effect of a magnetic field on Asparagus officinalis and Ocimum basilicum seed germination and seedling growth to be positive (Soltani et al. $2006 \mathrm{a}, \mathrm{b}$ ). The leaf, stem and root relative growth rates of tomato plants grown from magnetically treated seeds were greater than those of control plants (Souza et al. 2006). Magnetic field application enhanced chickpea seed germination speed, seedling length and seedling dry weight. Seeds were exposed in batches to static magnetic fields ranging in intervals of $50 \mathrm{mT}$ of from 0 to $250 \mathrm{mT}$ for $1-4 \mathrm{~h}$ in steps of 1 hour for all fields. Best results were obtained from exposures of $50 \mathrm{mT}$ for $2 \mathrm{~h}, 100 \mathrm{mT}$ for $1 \mathrm{~h}$ and $150 \mathrm{mT}$ for $2 \mathrm{~h}$ (Vashisth et al. 2008). Accelerated germination after magnetic stimulation of wheat seeds was observed working with 30, 45 and $60 \mathrm{mT}$ magnetic field strengths (Pietruszewski et al. 2010). 
In previous studies authors found an increase in the rate of germination of seeds and a stimulation of growth of seedlings. They found a positive growth response to a 125 $\mathrm{mT}$ and $250 \mathrm{mT}$ magnetic field in rice, wheat and barley seeds (Flórez et al. 2004; Martínez et al. 2000, 2007). The greatest stimulation of growth was observed in seeds chronically exposed to a magnetic field or in seeds treated for 24 hours. An increase of the initial growth stages and an early sprouting of maize seeds exposed to a stationary magnetic field was also observed (Flórez et al. 2007), treated plants grew higher and heavier than control. Effect of exposure of grass seeds to $125 \mathrm{mT}$ and $250 \mathrm{mT}$ was studied (Carbonell et al. 2008), finding that mean germination time was reduced by $10 \%$ compared with control seeds; the time required for germination onset was also reduced and the roots of grass seedlings from chronically exposed seeds grew higher and longer than those of untreated ones. Recently they have also obtained an early germination in Salvia officinalis L. and Calendula officinalis L, (Flórez, 2012).

Different theories have been proposed on the biological changes: external magnetic field effects at biochemical level and activating proteins and enzymes can increase the growth potential of seeds; magnetic field can interact with internal electric field of biological systems though its resonating behavior and cold be effective on the diffusion of biological particles in solutions (Liboff et al. 1988). The orientation of ferromagnetic particles and modulation of radical-pair reactions are reported as the mechanism of magnetic field effect (Faten et al. 2009). Living cells possess electric charges exerted by ions or free radicals, which act as endogenous magnets. Treatments with magnetic field are assumed to enhance seed vigor by influencing the biochemical processes that involve free radicals, and by stimulating activity of proteins and enzymes. Numerous studies suggested that magnetic field increases ions uptake and consequently improves nutrition value which could be a good alternative for chemical treatments (Stange et al. 2002). The influence of pre-sowing magnetic field treatments on tomato seed germination, seedling growth, emergence and plant growth parameters were studied. Seeds were exposed to $60 \mathrm{~Hz}$ induced by an electromagnet at 80,120, 160 and $200 \mathrm{mT}(\mathrm{rms})$ for $1-20 \mathrm{~min}$. An improved seed performance in terms of reduction of time required for the first seeds to complete germination, time to reach $50 \%$ germination, time between 10 and $90 \%$ germination with increasing germination rate, and increased germination percentage at 4 and 7 days, seedling shoot and root length compared to the untreated were found Souza et al. 2010). Results of the study of the effects of magnetic treatment of irrigation water and snow pea (Pisum sativum $\mathrm{L}$ ) and Kabuli chickpea (Cicer arietinum L) suggest that both magnetic treatment of seeds and magnetic treatment of irrigation water and seeds have the potential to improve the early seedling growth and nutrient contents of seedlings (Harsham et al. 2011). Revealing the relationships between MF and plant responses is becoming more and more important as new evidence reveals the ability of plants to perceive and respond quickly to varying MF by altering their gene expression and phenotype. The recent implications of MF reversal with plant evolution opens new horizons not only in plant science but also to the whole biosphere, from the simplest organisms to human beings (Maffei, 2014).

\section{CONCLUSIONS}

Results obtained in this study allow us to conclude that magnetic treatment improves germination rate of cereal seeds. In general, most of the parameters recorded for all the doses applied to maize, rice, barley and wheat seeds were better than control values. Thus, the rate of germination of treated seeds was higher than the untreated seed (C) rate. The greatest reductions in the mean germination time, the most relevant parameter, were for chronically exposure and $24 \mathrm{~h}$ to $125 \mathrm{mT}$. The early germination process are plotted in germination curves showing the left side displacement. Consequently, 125 $\mathrm{mT}$ magnetic field have a positive effect on cereal germination process.

\section{ACKNOWLEDGEMENTS}

The Technical University of Madrid (UPM) has supported this research, carried out by members of the research group "Investigation in Bioelectromagnetism Applied to Agroforestry Engineer", through the Project N.CCG07-UPM/0003348.

\section{REFERENCES}

[1] Aladjadjiyan, A. (2002). Study of the influence of magnetic field on some biological characteristics of Zea mais. Journal of Central European Agriculture, 2 (3), 89-94.

[2] Cakmak, T. Dumlupinar, R. Erdal, S. (2010). Acceleration of germination and early growth of wheat and bean seedlings grown under various magnetic field and osmotic conditions. Bioelectromagnetics, 2 (31), 120-129.

[3] Carbonell, V. Flórez, M. Martínez, E. Maqueda, R. Amaya, J.M. (2011). Study of stationary magnetic fields on initial growth of pea (Pisum sativum L.) Seeds. Seed Science and Technology 39, 673-679.

[4] Carbonell, M.V. Martínez, E. Flórez, M. Maqueda, R. López-pintor, A. Amaya, J.M. (2008). Magnetic field treatments improve germination and seedling growth 
in Festuca arundinacea Schreb. and Lolium perenne L. Seed Science and Technology, 36, 31-37.

[5] Faten, D. Jameel, M. Essam, H. (2009). Static Magnetic Field Influence on Elements Composition in Date Palm Phoenix dactylifera L. J. Agr. Biol. Sci 5, 161.

[6] Flórez, M (2004). Efecto de campos magnéticos estacionarios de $125 \mathrm{mT}$ y $250 \mathrm{mT}$ en la germinación de semillas y desarrollo de plántulas. Tesis doctoral. Escuela Técnica Superior de Ingenieros Agrónomos, Universidad Politécnica de Madrid.

[7] Flórez, M. Carbonell, M.V. Martínez, E. (2007). Exposure of maize seeds to stationary magnetic fields: effects on germination and early growth. Environmental and Experimental Botany, 1 (59) 6875.

[8] Flórez, M. Martínez, E. Carbonell, M.V. (2012). Effect of magnetic field treatment on germination of medicinal plants Salvia officinalis L. and Calendula officinalis L. Polish Journal of Environmental Studies, 1 (21) 57-63.

[9] García, F. Arza, L.I. (2001). Influence of a stationary magnetic field on water relations in lettuce seeds. Part I: Theoretical considerations. Bioelectromagnetics, 8(22) 589-595.

[10] Harsharn S. G. and Basant L. M. (2011). Magnetic treatment of irrigation water and snow pea and chickpea seeds enhances early growth and nutrient contents of seedlings. Bioelectromagnetics 32:58-65, 2011.

[11] ISTA. (2004). International Seed Testing Association. International Rules for Seed Testing. Seed Science and Technology, Zurich.

[12]Liboff, A.R. McLeod, B.R. (1998). Kinetics of channelized membrane ions in magnetic field Bioelectromagnetics 9, 39-51.

[13] Maffei, M.E. (2014). Magnetic field effects on plant growth, development, and evolution. Frontier Plant Science. http://dx.doi.org/10.3389/fpls.2014.00445

[14] Martínez, E. Carbonell, M.V. Amaya, J.M. (2000). Static magnetic field of $125 \mathrm{mT}$ stimulates the initial growth stages of barley (Hordeum vulgare L.). Electromagnetobiology, 3 (19) 271-277.

[15] Martínez, E. Carbonell, M.V. Flórez, M. (2002). Magnetic biostimulation of initial growth stages of wheat (Triticum aestivum, L.). Electromagnetic Biology and Medicine. 1 (21) 43-53.

[16] Phirke, P.S. Kubde, A.B.. Umbakar, S.P. (1996). The influence of magnetic field on plant growth. Seed Science and Technology, 24, 375-392.

[17] Pietruszewski, S. Kania, K. (2010). Effect of magnetic field on germination and yield of wheat. International Agrophysics, 24, 297-302.
[18] Podlesni, J. Pietruszewski, S. Podlesna, A. (2004). Efficiency of the magnetic treatment of broad bean seeds cultivated under experimental plot conditions. International Agrophysics, 1(18) 65-71.

[19] Shine, M.B. Guruprasad, K.N. Anand, A. (2011). Enhancement of germination, growth and photosynthesis in soybean by pre-treatment of seeds with magnetic field. Bioelectromagnetics, 6 (32) 474484.

[20] Soltani, F. Kashi, A. Arghavani, M. (2006a). Effect of magnetic field on Asparagus officinalis L. seed germination and seedling growth. Seed Science and Technology, 5 (34) 349-353.

[21] Soltani, F. Kashi, A. Arghavani, M. (2006b). Effect of magnetic field on Ocimum basilicum seed germination and seedling growth. Acta Horticulturae, 723, 279282.

[22] Souza, A. García, D. Sueiro, L. Gilart, F Porras, E. Licea, L. (2006). Pre-sowing magnetic treatments of

tomato seeds increase the growth and yield of plants. Bioelectromagnetics, 4, 27, 247-257.

[23] Souza, A.; Sueiro, L.; García, D.; Porras, E. (2010). Extremely low frequency non-uniform magnetic fields improve tomato seed germination and early seedling growth. Seed Science and Technology, 38, 1. 61-72.

[24] Stange, B.C. Rowlans, R.E. Rapley, B.I. J.V. Podd, B.I. (2002). ELF magnetic fields increase aminoacid uptake into Vicia faba L. roots and alter ion movement across the plasma membrane. Bioelectromagnetics 23, 347-354.

[25] Vashisth, A. Nagarajan, N. (2008). Exposure of seeds to static magnetic field enhances germination and early growth characteristics in chickpea. Bioelectromagnetics, 7 (29) 571-578.

[26] Yano, A. Hidaka, E. Fujiwara, K. Iimoto, M. (2001).Induction of primary root curvature in radish seedlings in a static magnetic field. Bio electromagnetics, 